\title{
Galactic interstellar extinction: Neckel \& Klare view and beyond
}

\author{
A. C. Robin
}

\begin{abstract}
Institut Utinam, CNRS UMR6213, Université de Franche-Comté, Observatoire de Besançon, Besançon, France
\end{abstract}
e-mail: annie.robin@obs-besancon.fr

Interstellar extinction can be seen either as a veil that prevents anything being seen in depth in the Galactic plane or as a tracer of interstellar matter, an important component in Galaxy evolution. Stars are good tracers of the extinction when their reddening and their absolute magnitude can be estimated from their observable properties. The main problem arising when studying extinction comes from the patchiness of the extinction. Therefore a sufficiently dense stellar mesh is needed. The second problem is to accurately determine distances to the clouds. Before the famous Neckel \& Klare (1980) paper $^{1}$, several attempts had been made to trace the extinction all around the sky and in depth. Based on photoelectric observations of a limited number of stars, these attempts allowed the extinction to be traced, but was limited in spatial extent (the Galactic plane), in spatial resolution (a few stars per square degree at maximum), and in distance (1-2 kpc).

Following their first extinction study from 4700 stars (Neckel 1966), Klare \& Neckel undertook a considerable observing program to obtain new OB star measurements in the Southern Milky Way. They performed observations from 1966 to 1975 in South Africa of 1660 stars with objective prisms and photoelectric photometers (Klare \& Szeidl 1966). Neckel \& Klare built their extinction maps using these observations, together with a wide number of others, mainly photoelectric UBV, MK spectral types, and $\mathrm{H} \beta$ measurements for more than $11000 \mathrm{O}$ to $\mathrm{F}$ stars. The absolute magnitude error $(0.46 \mathrm{mag})$ translates into an error of $25 \%$ on individual distances, while the error on the extinction Av is estimated at 0.1-0.2 mag. Determining the extinction as a function of distance is not independent of the determination of the extinction law. Neckel \& Klare used a ratio of total-to-selective extinction $R_{\mathrm{V}}$ of 3.1, a value still generally used, while it is known to deviate from this value in fields with high extinction. This is probably not a major problem in the Neckel \& Klare studies, since the limitation comes mainly from the spatial resolution of the map.

The analysis covered $|b|<7^{\circ}$ at maximum. Neckel \& Klare mentioned that the result is biased towards lower extinction at a large distance because surveys are incomplete for highly obscured stars. Because the bias is higher when the dispersion in

1 It should be noted that this paper is sometimes wrongly cited as Neckel, Klare, and Sarcander, due to a mistake in the NASA ADS data base. The reference Neckel et al. (1980) refers to the catalog of extinction data of the 12547 stars and open clusters published in the Bulletin $d u C D S$, while Neckel \& Klare published in A\&A contains the analysis and the extinction maps.
Av is higher, they chose the 325 regions to be as homogeneous as possible to minimize this effect.

The distribution in distance was limited by the star detection. Extinction was generally determined up to $5 \mathrm{kpc}$, in a few cases up to $9 \mathrm{kpc}$, but sometimes only $2 \mathrm{kpc}$. Neckel \& Klare mapped the distribution of clouds in the solar vicinity and identified a list of clouds. They noticed a hole in the cloud distribution near the Sun: at longitude $180^{\circ}$ and $270^{\circ}$ there are almost no clouds in the plane until a few hundred parsecs, a feature later confirmed by Gaustad et al. (1991) and others. They estimated the list of clouds to be complete to about $1 \mathrm{kpc}$. Their analysis terminated with the statement that the distribution of clouds does not correlate well with known spiral features in the solar neighborhood.

A considerable number of extinction studies can be found in the literature, a few of which are cited here. They are based on different tracers. Some allow determination of the integrated extinction up to the edge of the Galaxy and give 2D maps: HI and galaxy counts (Burstein \& Heiles 1978, 1982), galaxy colors (Cambresy et al. 2005), FIR emission (Schlegel et al. 1998). Some map the extinction at a given distance (Schultheis et al. 1999; Cambresy 2002). Others are improvements on the Neckel $\&$ Klare method to determine the extinction as a function of distance from photometric or spectroscopic stellar samples and to lead to 3D maps (Arenou et al. 1992). Still others attempt to turn extinction maps into models (Wainscoat et al. 1992; Hakkila et al. 1997; Mendez \& Van Altena 1998; Drimmel \& Spergel 2001; Amores \& Lépine 2005).

These studies provide either 2D or 3D maps, compromising between having a high spatial resolution on the projected sky or getting information in 3D when distance estimators are available. The latter is often at the detriment of resolution, but is more powerful for understanding the structure of the ISM. Since the extinction can vary on very small scales (arc minute or smaller) and present filamentary structures, probably on a scale of tens of $\mathrm{AU}$, the extinction is known with a high level of detail only at short distances. Another important difference between 2D and $3 \mathrm{D}$ maps is that 2D maps determined from integrated IR flux or using external galaxies as tracers give the true total extinction, while 3D maps give the distribution of the extinction up to a distance and suffer from the bias mentioned by Neckel \& Klare: they underestimate the extinction by lacking completeness where the extinction is high and patchy.

In spite of these defects and imperfections, 3D maps have been proved to be very useful and are abundantly used. Those by Neckel $\&$ Klare are used to estimate the extinction in clouds, 
in front of clusters, or in other interesting galactic objects, even in extragalactic studies ${ }^{2}$.

More sophisticated methods are nowadays proposed to recover the extinction along the line of sight and trace the 3D distribution. In general they use sophisticated ways of estimating distances from the photometry.

Drimmel et al. (2003) propose a method of producing a 3D model of the extinction, partly based on the Drimmel \& Spergel (2001) model and on the method by Lopez-Corredoira et al. (2002), which mapped the extinction by extracting the redclump population from the 2MASS color-magnitude diagrams. Marshall et al. (2006) assume a smooth population synthesis model (Robin et al. 2003) and fit the extinction to recover the distribution in color-magnitude diagrams from 2MASS survey. They reach a resolution of 15 armin and a few hundred pc in distance. Sale et al. (2009) propose to use IPHAS photometry to trace the extinction. Using stellar models at solar metallicity, they expect to get a resolution of 10 arcmin and $100 \mathrm{pc}$ in distance. The method looks promising but has not yet been extensively used or compared with other studies.

The comparison of results obtained by these three methods shows a variety of situations depending on the field, but without any conspicuous systematics. Typically, the differences in Av at a given distance from one model to another are on the order of $20 \%$. Several reasons can be called upon: (i) different extinction methods trace different phases of the interstellar matter. They are sensitive to different grain sizes, temperatures, and gas/dust ratios; (ii) maps are done at different spatial resolutions. Since the dust distribution is highly inhomogeneous and clumpy down to small scales, the mean extinction varies as a function of the resolution considered; (iii) the $R_{V}$ varies from one line of sight to the other, while it is often taken as a constant, and the assumed extinction law varies from one model to the next; (iv) the results sometimes depend on assumptions about the geometry of the disk and the spiral arms which might not be correct, or too simplified.

The 3D mapping of the extinction has led to tracing the distribution of the interstellar medium (ISM). The disk appears thin and flared, but also probably slightly inclined with regards to the stellar mid-plane (Pandey \& Mahra 1987; Joshi 2005). Neckel $\&$ Klare pointed out that the clouds near the Sun do not seem to follow any typical spiral structure, because their sample is too limited in distance. The spiral stucture in the dust is now proven by Marshall et al. (2008), Amores \& Lépine (2005), or Drimmel \& Spergel (2001). Despite the patchiness, it generally appears that gas, molecular clouds, and dust follow some kind of spiral structure, as do young stellar populations, but the uncertainty on distances renders the layout of the arms difficult.

In the same spirit as the Neckel \& Klare study, important steps will be taken in the future by using the large-scale surveys, applying the methods of analysis proposed above, and sophisticated ways of estimating distances. The choice of the wavelength to measure the extinction is critical. Maps at high latitudes or low extinction are worth being done with visible data that are more sensitive to low extinction, but from the infrared in regions of high extinction; hence, a full 3D map would require a combination of visible and infrared surveys.

In a promising future, Gaia will advance two steps closer to determining extinction, thanks to the accurate photometry and distances from trigonometric parallaxes. As a first step, it will allow the interstellar extinction to be determined at an unprecedented accuracy of $0.05 \mathrm{mag}$, and distances at better than $1 \%$. This will feature the local extinction up to about $2 \mathrm{kpc}$ with a resolution in cloud size of less than about 10-20 pc over the whole sky. The second step will be made using the photometer BP/RP accurate to a few milli-magnitudes. Gaia will thus provide a huge database for mapping the extinction at a spatial resolution never obtained before, to very large distances (accurate at 10\% up to $10 \mathrm{kpc}$ ), but with a limitation in regions of high extinction where Gaia could be complemented by near-infrared surveys. Besides the comparison between Gaia distances and extinction maps, infrared emission from Herschel will revolutionize our view of the ISM, drawing a consistent 3D picture of the interstellar medium and its physical properties, and this is a tremendous advance in the quest initialized by the pioneering work of Neckel \& Klare.

\section{References}

Amores, E. B., \& Lépine, J. R. D. 2005, AJ, 130, 659

Arenou, F., Grenon, M., \& Gomez, A. 1992, A\&A, 258, 104

Burstein, D., \& Heiles, C. 1978, ApJ, 225, 40

Burstein, D., \& Heiles, C. 1982, AJ, 87, 1165

Cambrésy, L., Beichman, C. A., Jarrett, T. H., \& Cutri, R. M. 2002, AJ, 123, 2559

Cambrésy, L., Jarrett, T. H., \& Beichman, C. A. 2005, A\&A, 435, 131

Drimmel, R., \& Spergel, D. N. 2001, ApJ, 556, 181

Drimmel, R., Cabrera-Lavers, A., \& Lopez-Corredoira, M. 2003, A\&A, 409, 205

Gaustad, J., Alford, M., MacKenzie, M., et al. 1991, BA\&AS, 23, 1365

Joshi, Y. C. 2005, MNRAS, 362, 1259

Hakkila, J., Myers, J. M., Stidham, B. J., \& Hartmann, D. H. 1997, AJ, 114, 2043

Klare, G., \& Szeidl, B. 1966, Veroffentl. Landessternwarte HeidelbergKonigstuhl, 18

Klare, G., \& Neckel, T. 1977, A\&AS, 27, 215

López-Corredoira, M., Cabrera-Lavers, A., Garzón, F., \& Hammersley, P. L. 2002, A\&A, 394, 883

Marshall, D. J., Robin A. C., Reylé, C., Schultheis, M., \& Picaud, S. 2006, A\&A, 453,635

Marshall, D., Robin, A. C., \& Reylé, C. 2008, BA\&AS 40, 213

Mendez, R. A., \& van Altena, W. F. 1998, A\&A, 330, 910

Neckel, T. 1966, Z. Astrophys., 63, 221

Neckel, Th., \& Klare, G. 1980, A\&AS, 42, 251

Neckel, Th., Klare, G., \& Sarcander, M. 1980, BICDS, 19, 61

Pandey, A. K., \& Mahra, H. S. 1987, MNRAS, 226, 635

Robin, A. C., Reylé, C., Derrière, S., \& Picaud, S. 2003, A\&A, 409, 523

Sale, S. E., Drew, J. E., Unruh, Y. C., et al. 2009, MNRAS, 392, 497

Schlegel, D. J., Finkbeiner, D. P., \& Davis, M. 1998, ApJ, 500, 525

Schultheis, M., Ganesh, S., Simon, G., et al. 1999, A\&A, 349, L69

Wainscoat, R. J., Cohen, M., Volk, K., Walker, H. J., \& Schwartz, D. E. 1992, ApJS, 83,111

\footnotetext{
2 At that time, extragalactic studies more generally used Burstein \& Heiles (1978) 2D maps, based on HI and galaxy counts. This paper has been cited more due to the regions covered: Burstein \& Heiles (1978) maps cover the high latitudes, while Neckel \& Klare do not.
} 